GT2004-53913

\title{
SUBSURFACE STRESS FIELDS IN FCC SINGLE CRYSTAL ANISOTROPIC CONTACTS
}

\author{
Nagaraj K. Arakere ${ }^{1}$ \\ Associate Professor \\ Mechanical \& Aerospace Engineering \\ University of Florida \\ Gainesville, FL 32611-6300
}

\author{
Erik Knudsen \\ Graduate Student \\ Mechanical \& Aerospace Engineering \\ University of Florida \\ Gainesville, FL 32611-6300
}

\author{
Gregory R. Swanson, Ph.D \\ NASA Marshall Space Flight Center \\ ED 22, Structural Miechanics Group \\ Huntsville, Alabama \\ Greg.Swanson@nasa.gov
}

\author{
Gregory Duke \\ Mechanical Engineer \\ JE Sverdrup \\ Huntsville, Alabama \\ Greg.Duke@msfc.nasa.gov
}

\author{
Gilda Ham-Battista, Ph.D \\ Mechanical Engineer \\ ERC, inc. \\ Huntsville, Alabama \\ Gilda.Battista@msfc.nasa.gov
}

\begin{abstract}
Single crystal superalloy turbine blades used in high pressure turbomachinery are subject to conditions of high temperature, triaxial steady and alternating stresses, fretting stresses in the blade attachment and damper contact locations, and exposure to high-pressure hydrogen. The blades are also subjected to extreme variations in temperature during start-up and shutdown transients. The most prevalent high cycle fatigue (HCF) failure modes observed in these blades during operation include crystallographic crack initiation/propagation on octahedral planes, and noncrystallographic initiation with crystallographic growth. Numerous cases of crack initiation and crack propagation at the blade leading edge tip, blade attachment regions, and damper contact locations have been documented. Understanding crack initiation/propagation under mixed-mode loading conditions is critical for establishing a systematic procedure for evaluating HCF life of single crystal turbine blades.
\end{abstract}

This paper presents analytical and numerical techniques for evaluating two and three dimensional subsurface stress fields in anisotropic contacts. The subsurface stress results are required for evaluating contact fatigue life at damper contacts and dovetail attachment regions in single crystal nickel-base superalloy turbine blades. An analytical procedure is presented for evaluating the subsurface stresses in the elastic half-space, based on the adaptation of a stress function method outlined by
Lekhnitskii [1]. Numerical results are presented for cylindrical and spherical anisotropic contacts, using finite element analysis (FEA). Effects of crystal orientation on stress response and fatigue life are examined. Obtaining accurate subsurface stress results for anisotropic single crystal contact problems require extremely refined three-dimensional (3-D) finite element grids, especially in the edge of contact region. Obtaining resolved shear stresses (RSS) on the principal slip planes also involves considerable post-processing work. For these reasons it is very advantageous to develop analytical solution schemes for subsurface stresses, whenever possible.

\section{INTRODUCTION}

Single crystal nickel base superalloy turbine blades are especially prone to fretting/contact fatigue damage because the subsurface shear stresses induced by fretting action at the damper contact and blade attachment regions can result in crystallographic initiation and crack growth along octahedral planes. The presence of fretting in conjunction with a mean stress in the body of a component can lead to a marked reduction in HCF life, sometimes by a factor as great as $10[2$, 3]. Fretting occurs when assemblies of components such as blade and disk attachment surfaces, bolt flanges, snap fit areas, and other clamped members are subjected to vibration, resulting in contact damage. The combined effects of

Corresponding author 
corrosion, wear, and fatigue phenomena at the fretting contact facilitate the initiation and subsequent growth of cracks.

Currently the most widely used single crystal nickel base turbine blade superalloys are PWA 1480, PWA 1484, RENE' $\mathrm{N}-5$ and CMSX-4. These alloys play an important role in commercial, military and space propulsion systems [5-8]. Military gas turbine mission profiles are characterized by multiple throttle excursions associated with maneuvers such as climb, intercept and air-to-air combat. This mission shifts attention to fatigue and fracture considerations associated with areas below the blade platform which contain various stress risers in the form of buttresses and attachments. Blade-disk attachment areas and blade frictional damping devices are particularly prone to fretting/galling fatigue damage [5]. Rocket engine service presents another set of requirements that shifts emphasis to low temperature fatigue and fracture capability with particular attention given to environmental effects (i.e. high pressure hydrogen gas exposure, thermal, and cryogenic). Attention has shifted from oxidation erosion, creep, stress rupture and creep fatigue damage mechanisms to the micromechanics of fatigue and fracture observed between room temperature and $871 \mathrm{C}(1600 \mathrm{~F})$. Fatigue crack initiation, threshold, and Region II fatigue crack growth are of primary importance and the demand for improvements in fracture mechanics properties for turbine blade alloys is imminent [5].

Study of crack initiation under mixed mode loading is important for understanding fretting fatigue crack initiation in single crystals. The subsurface shear stresses induced by fretting action can result in crystallographic initiation of failure, under mixed mode loading conditions. Fretting fatigue at low slip amplitudes that induces little or no surface damage can result in greatly reduced fatigue life with accelerated subsurface crystallographic crack initiation, akin to subsurface shear stress induced rolling bearing fatigue. The complex interaction between the effects of environment and stress intensity determines which point-source defect species initiates a crystallographic or noncrystallographic fatigue crack [5-8].

This paper presents analytical and numerical methods to evaluate the subsurface stresses in face-centered cubic (FCC) single crystal cylindrical and spherical contacts as a function of crystallographic orientation, and contact loads. The subsurface stresses evaluated are subsequently used to assess contact fatigue life, based on a fatigue life model developed previously [9-12]. The motivation for this work is provided by the crystallographic subsurface cracks induced at the damper contact locations in single crystal turbine blades. Figure 1 shows a schematic of the damper contact location. Figure 2 shows a close-up view of the subsurface induced crystallographic crack propagating on intersecting octahedral planes, ultimately resulting in a pyramidal hole in the blade platform [13].

There is a considerable body of work done on fretting fatigue damage of isotropic polycrystalline materials. Some representative examples are by Hills and Nowell [2], Giannokopoulos and Suresh [14], Swolwinsky and Farris [15], Attia and Waterhouse [16], Hoeppner [17], Vingsbo and Soderberg [18], and Ruiz, et al [19]. However, studies on subsurface contact stresses and mechanics of fretting fatigue crack initiation and crack growth in orthotropic single crystal materials are very few. There is an extensive body of literature available in the classical area on the evaluation of subsurface stresses for nonconformal contacts in isotropic materials using analytical methods [20]. However, the amount of published literature involving analytical solutions in anisotropic nonconformal contacts is considerably less. Green and Zerna [21] looked at the 2D anisotropic contact problem in 1954, for a specific type of anisotropy. Willis [22] examined the Hertzian elliptical contact problem for anisotropic half-spaces using a Fourier transform method. Turner [23] examined the spherical contact between transversely isotropic nonconformal bodies. Fan and Keer [24] examine the 2D contact problem using the analytic function continuation approach based on the Stroh formulation [25]. Vlassak, et al [26] looked at calculating an

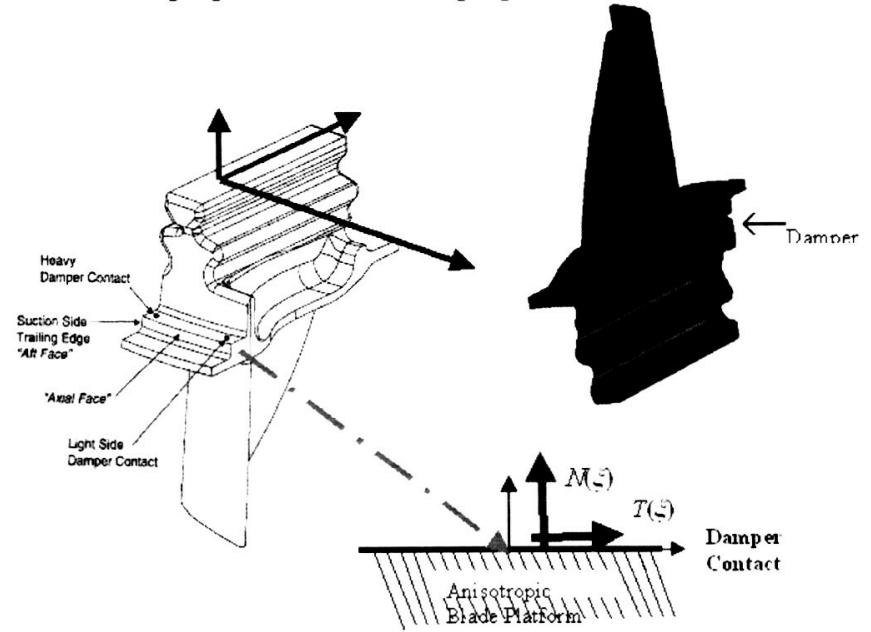

Figure 1. Damper contact locations on the turbine blade
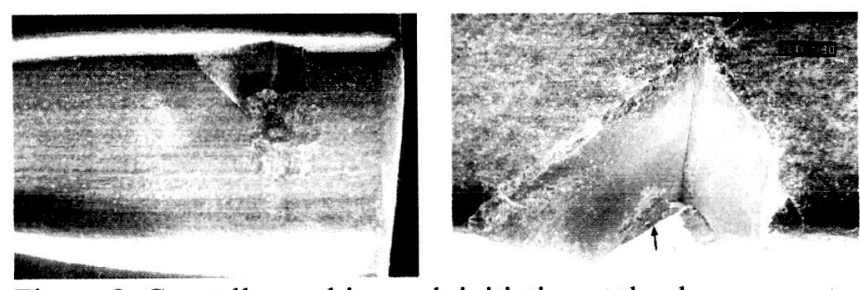

Figure 2. Crystallographic crack initiation at the damper contact location shown in Fig. 1 [13]

effective indentation modulus for anisotropic contacts. Analytical solutions were developed for indenters of arbitrary shape being pressed into an anisotropic half-space. For axisymmetric indenters, a limited family of Green's functions is used to obtain a solution for the displacement field. This solution is denoted as an equivalent isotropic solution.

In this paper, we present an analytical solution for a cylindrical contact, using the stress function approach outlined by Lekhnitskii [1], for an anisotropic half space under conditions of generalized plane strain problem. Finite element subsurface stress results of the cylindrical and spherical anisotropic contacts modeling the damper contact locations shown in Figure 1 are also presented. 


\section{NOMENCLATURE}

Deformation Mechanisms and Elastic Anisotropy in FCC Single Crystals.

Nickel based single-crystal materials are precipitationstrengthened, cast, mono-grain superalloys based on the $\mathrm{Ni}-\mathrm{Cr}$ Al system. The microstructure consists of approximately $60 \%$ to $70 \%$ by volume of $\gamma^{\prime}$ precipitates in a $\gamma$ matrix. The $\gamma^{\prime}$ precipitate, based on the intermetallic compound $\mathrm{Ni}_{3} \mathrm{Al}$, is the strengthening phase in nickel-base superalloys and is a Face Centered Cubic (FCC) structure. The $\gamma^{\prime}$ precipitate suspended within the $\gamma$ matrix also has a FCC structure and is comprised of nickel with cobalt, chromium, tungsten and tantalum in solution [5].

Deformation mechanisms in single crystals are primarily dependent on microstructure, orientation, temperature, and crystal structure. The operation of structures at high temperature places additional materials constraints on the design that are not required for systems that operate at or near room temperatures. In general, materials become weaker with increasing temperature due to thermally activated processes, such as multiple slip and cross-slip. At temperatures in excess of approximately half the homologous temperature (the ratio of the test temperature to the melting point, $=\mathrm{T} / \mathrm{T}_{\mathrm{m}}$ ), diffusion controlled processes (e.g., recovery, recrystallization, dislocation climb and grain growth) become important, which results in further reductions in strength. Slip in metal crystals often occurs on planes of high atomic density in closely packed directions. The four octahedral planes corresponding to the high-density planes in the FCC crystal have three primary slip directions (easy-slip) resulting in twelve independent primary $<110>\{111\}$ slip systems. The four octahedral slip planes also have three secondary slip directions resulting in twelve independent secondary $<112>\{111\}$ slip systems. In addition, the three cube slip planes have two slip directions resulting in six independent $<110>\{100\}$ cube slip systems. Thus there are twelve primary and twelve secondary slip systems associated with the four octahedral planes and six cube slip systems with the three cube planes, for a total of thirty slip systems [27]. At high temperatures, slip has been observed in non-close-pack directions on the octahedral plane, and on the cube plane, in FCC crystals.

Elastic response of FCC crystals is obtained by expressing Hooke's law for materials with cubic symmetry. The generalized Hooke's law for a homogeneous anisotropic body in Cartesian coordinates $(\mathrm{x}, \mathrm{y}, \mathrm{z}$ with origin at point $\mathrm{O})$ is given by Eq. (1) $[1,27]$.

$$
\{\varepsilon\}=\left\lfloor a_{i j}\right\rfloor\{\sigma\}
$$

$\left[a_{i j}\right]$ is the matrix of 36 elastic coefficients, of which only 21 are independent, since $\left[\boldsymbol{a}_{i j}\right]=\left[\boldsymbol{a}_{\mathrm{ji}}\right]$. The elastic properties of FCC crystals exhibit cubic symmetry, also described as cubic syngony. The elastic properties of materials with cubic symmetry can be described with three independent constants designated as the elastic modulus, shear modulus, and Poisson ratio [1] and hence [ $\left.\boldsymbol{a}_{i j}\right]$ can be expressed as shown in Eq. (2), in the material coordinate system (FCC crystal axes are parallel to $\mathrm{x}, \mathrm{y}$ and $\mathrm{z}$ coordinate axes). In contrast to the FCC single crystal material, an isotropic material can only have two independent elastic constants.

$$
\left[a_{j}\right]=\left[\begin{array}{cccccc}
a_{11} & a_{12} & a_{12} & 0 & 0 & 0 \\
a_{12} & a_{12} & a_{12} & 0 & 0 & 0 \\
a_{12} & a_{12} & a_{11} & 0 & 0 & 0 \\
0 & 0 & 0 & a_{44} & 0 & 0 \\
0 & 0 & 0 & 0 & a_{44} & 0 \\
0 & 0 & 0 & 0 & 0 & a_{44}
\end{array}\right], a_{11}=\frac{1}{E_{x}}, a_{44}=\frac{1}{G_{y z}}, a_{12}=-\frac{\nu_{y z}}{E_{x x}}=-\frac{\nu_{p y}}{E_{p y}}
$$

The elastic constants in the generalized Hooke's law of an anisotropic body, $\left[a_{\mathrm{ij}}\right]$, vary with the direction of the coordinate axes. For orientations other than the $(x, y, z)$ axes, the $\left[a_{i j}\right]$ matrix varies with the crystal orientation. In the case of an isotropic body the constants are invariant in any orthogonal coordinate system. Consider a Cartesian coordinate system ( $x$ ', $\left.y^{\prime}, z^{\prime}\right)$ that has rotated about the origin $O$ of $(x, y, z)$. The elastic constant matrix $\left[a^{\prime}{ }_{i j}\right]$ in the $\left(x^{\prime}, y^{\prime}, z^{\prime}\right)$ coordinate system that relates $\left\{\varepsilon^{\ominus}\right\}$ and $\left\{\sigma^{*}\right\} \quad\left(\left\{\varepsilon^{\ni}\right\}=\left[a_{i j}\right]\left\{\sigma^{\ni}\right\}\right)$ is given by the following transformation [1].

$$
\left[\boldsymbol{\alpha}_{i j}^{i}\right]=[\boldsymbol{Q}\}^{r}\left[\boldsymbol{\alpha}_{i j}\right][\boldsymbol{Q}]=\sum_{m=1}^{6} \sum_{x=1}^{6} a_{m x} \ell_{m i} \ell_{x i},(i, j=1,2, \ldots, 6)
$$

The transformation matrix $[Q]$ is a $6 \times 6$ matrix that is a function of the direction cosines between the $(x, y, z)$ and $\left(x^{\prime}, y^{\prime}, z^{\prime}\right)$ coordinate axes. Knowing the state of stress at a given location, in the material coordinate system $(x, y, z)$, the resolved shear stresses on the twelve primary octahedral slip systems, denoted by $\tau^{1}, \tau^{2}, \ldots, \tau^{12}$, can be readily obtained using the transformation given by Eq. (4) [30]. The slip plane and slip direction of the twelve primary octahedral slip systems are given in Table 1 [27]. The resolved shear stresses on the secondary octahedral and cube planes are obtained using similar expressions $[9,27]$.

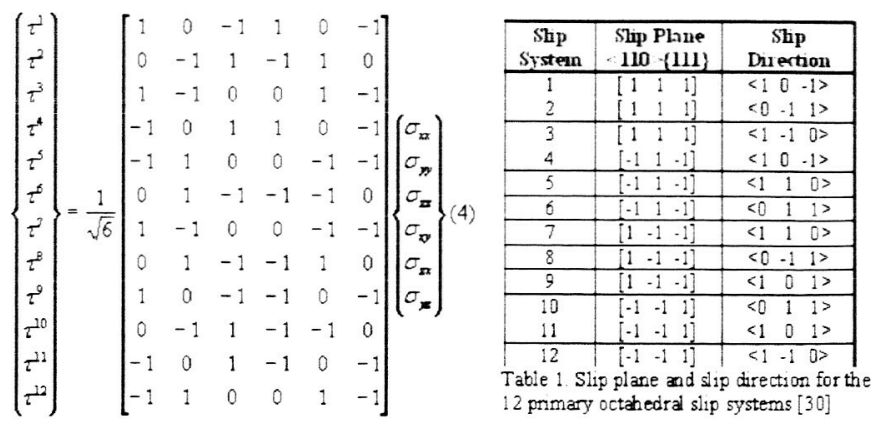

\section{Analytical Solution for the Two-Dimensional Stress Distribution (Generalized Plane Deformation) in an Anisotropic Elastic Half-Space}

The damper contact regions shown in Figure 1 will be modeled as an elastic anisotropic half-space. This approximation is reasonable since Hertzian type contact stresses are confined to very small volumes in the vicinity of the contact. An analytical procedure will be presented for evaluating the subsurface stresses in the elastic half-space using a stress function 
approach outlined by Lekhnitskii [1]. Lekhnitskii's method for a general anisotropic body has been adapted for a orthotropic FCC single crystal half space. Figure 3 shows the elastic halfspace subjected to normal traction $N(\xi)$ and tangential traction $T(\xi)$ over the region $-\mathrm{a}$ to $+\mathrm{a}$ on the $\mathrm{x}$-axis. The traction forces are independent of $z$, and functions of $x$ and $y$ only. The stresses are also functions of $\mathrm{x}$ and $\mathrm{y}$ only.

The equilibrium equations under generalized plane strain conditions, for an anisotropic halfspace, are expressed as follows [1]:

$$
\begin{aligned}
& \frac{\partial \sigma_{x}}{\partial x}+\frac{\partial \tau_{x y}}{\partial y}=0 \\
& \frac{\partial \sigma_{y}}{\partial y}+\frac{\partial \tau_{x y}}{\partial x}=0 \\
& \frac{\partial \tau_{x z}}{\partial x}+\frac{\partial \tau_{y z}}{\partial y}=0
\end{aligned}
$$

Note that the third equilibrium equation in Eqs. (5) is not used for plane strain condition for isotropic materials. However, because of shear coupling induced by anisotropy, the shear stresses $\tau_{\mathrm{xz}}$ and $\tau_{\mathrm{yz}}$ are nonzero, and are functions of $x$ and $y$.

The stress-strain relations, as defined by the Hooke's law, Eqs. (1), are given by

$$
\begin{aligned}
& \varepsilon_{x}=a_{11} \sigma_{x}+a_{12} \sigma_{y}+\ldots .+a_{16} \tau_{x y} \\
& \varepsilon_{y}=a_{12} \sigma_{x}+a_{22} \sigma_{y}+\ldots .+a_{26} \tau_{x y} \\
& . \\
& \gamma_{x y}=a_{16} \sigma_{x}+a_{26} \sigma_{y}+\ldots .+a_{66} \tau_{x y}
\end{aligned}
$$

where $\left[\boldsymbol{a}_{\mathrm{ij}}\right]$ are a function of crystallographic orientation.

Under the assumptions of generalized plane strain, the subsurface stresses due to the applied traction forces can be determined as outlined below.

The stress functions are given by

$$
\begin{aligned}
& \dot{\phi_{1}^{\prime}}(z)+\dot{\phi}_{2}^{\prime}(z)+\lambda_{3} \dot{\phi}_{3}^{\prime}(z)=-\frac{1}{2 \pi i} \int_{-a}^{+a} \frac{N(\xi)}{\xi-z} d \xi \\
& \mu_{1} \dot{\phi}_{1}^{\prime}(z)+\mu_{2} \phi_{2}^{\prime}(z)+\mu_{3} \lambda_{3} \phi_{3}^{\prime}(z)=-\frac{1}{2 \pi i} \int_{-a}^{+a} \frac{T(\xi)}{\xi-z} d \xi \\
& \lambda_{1} \phi_{1}^{\prime}(z)+\lambda_{2} \phi_{2}^{\prime}(z)+\dot{\phi}_{3}^{\prime}(z)=0
\end{aligned}
$$

The $\mu_{\mathrm{i}}$ are the roots of the cylindrical characteristic equation, given by Eq. (10), and $z=x+\mu y$.

$$
l_{4}(\mu) \cdot l_{2}(\mu)-l_{3}^{2}(\mu)=0
$$

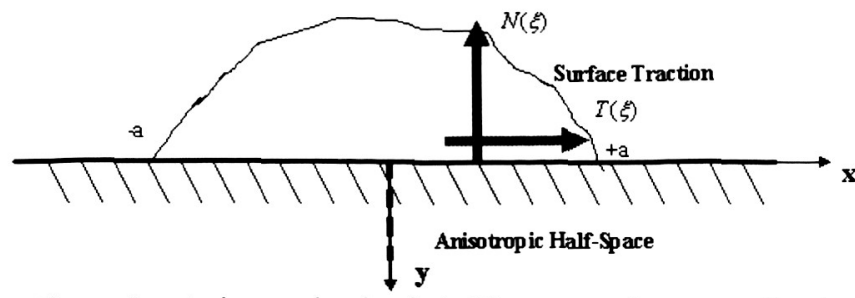

Figure 3. Anisotropic elastic half-space under generalized plane deformation subjected to normal and tangential traction forces.

$$
\begin{aligned}
& l_{2}(\mu)=\beta_{55} \mu^{2}-2 \beta_{45} \mu+\beta_{55} ; \quad l_{3}(\mu)=\beta_{15} \mu^{3}-\left(\beta_{14}+\beta_{56}\right) \mu^{2}+\left(\beta_{25}+\beta_{46}\right) \mu-\beta_{24} \\
& l_{4}(\mu)=\beta_{11} \mu^{4}-2 \beta_{16} \mu^{3}+\left(2 \beta_{12}+\beta_{66}\right) \mu^{2}-2 \beta_{26} \mu+\beta_{22}, \quad \beta_{i j}=a_{i j}-\frac{a_{13} a_{33}}{a_{33}} \\
& \lambda_{1}=-\frac{l_{3}\left(\mu_{1}\right)}{l_{2}\left(\mu_{1}\right)}, \lambda_{2}=-\frac{l_{3}\left(\mu_{2}\right)}{l_{2}\left(\mu_{2}\right)}, \lambda_{3}=-\frac{l_{3}\left(\mu_{3}\right)}{l_{4}\left(\mu_{3}\right)}
\end{aligned}
$$

The matrix $a_{\mathrm{ij}}$ relates the strains to the stresses. The $a_{\mathrm{ij}}$ are functions of the crystal orientation.

The stresses are then given by:

$$
\begin{aligned}
\sigma_{x} & =2 \operatorname{Re}\left[\mu_{1}^{2} \phi_{1}^{\prime}(z)+\mu_{2}^{2} \phi_{2}^{\prime}(z)+\mu_{3}^{2} \lambda_{3} \phi_{3}^{\prime}(z)\right] \\
\sigma_{y} & =2 \operatorname{Re}\left[\phi_{1}^{\prime}(z)+\phi_{2}^{\prime}(z)+\lambda_{3} \phi_{3}^{\prime}(z)\right] \\
\tau_{x y} & =-2 \operatorname{Re}\left[\mu_{1} \phi_{1}^{\prime}(z)+\mu_{2} \dot{\phi}_{2}^{\prime}(z)+\mu_{3} \lambda_{3} \phi_{3}^{\prime}(z)\right] \\
\tau_{x z} & =2 \operatorname{Re}\left[\mu_{1} \lambda_{1} \phi_{1}^{\prime}(z)+\mu_{2} \lambda_{2} \dot{\phi}_{2}^{\prime}(z)+\mu_{3} \phi_{3}^{\prime}(z)\right] \\
\tau_{y z} & =-2 \operatorname{Re}\left[\lambda_{1} \phi_{1}^{\prime}(z)+\lambda_{2} \phi_{2}^{\prime}(z)+\phi_{3}^{\prime}(z)\right] \\
\sigma_{z} & =-\frac{1}{a_{33}}\left[a_{13} \sigma_{x}+a_{23} \sigma_{y}+a_{34}{ }^{\tau} y z+a_{35^{\tau} x z}+a_{36^{\tau} x y}{ }^{\tau}\right] \\
z_{i} & =x+\mu_{i} y
\end{aligned}
$$

The normal traction force $N(\xi)$ used is the Hertzian cylindrical contact pressure as $N(\xi)=p_{o} \sqrt{1-\xi^{2} / a^{2}}$ and $T(\xi)=\mu_{f} p_{o} \sqrt{1-\xi^{2} / a^{2}}$, where $p_{\mathrm{o}}$ is the peak pressure and $\mu_{\mathrm{f}}$ the coefficient of friction. The tangential traction force is based on a sliding contact and not a contact in partial slip. It must be noted that we are not solving a contact problem here, but rather a stress analysis problem in an elastic anisotropic half space subject to normal and tangential traction forces. The contact dimensions are obtained from a simulated Hertzian cylindrical contact. The semi-elliptical normal pressure distribution seen in isotropic cylindrical contacts is also true for anisotropic contacts [12]. The stress solution has been programmed and subsurface stresses computed for various crystal orientations.

Figure 4 shows the finite element model used for the numerical results. This ANSYS model represents an elastic anisotropic half-space and was developed using 8-node hexahedral elements (SOLID45's) in the contact reg(8h and 4-node tetrahedral elements (SOLID45's) in the far field. The load functions, $N(\xi)=p_{o} \sqrt{1-\xi^{2} / a^{2}}$ and $T(\xi)=\mu_{f} p_{o} \sqrt{1-\xi^{2} / a^{2}}$ were applied directly to the finite element model. This applied stress problem does not require the use of contact elements.

Figure 5 shows a comparison of the analytical and finite element $\sigma_{\mathrm{y}}$ stress fields for $a=0.01 \mathrm{inch}, p_{\mathrm{o}}=260 \mathrm{ksi}$, and for the $(x, y, z)$ axes parallel to the edges of the FCC crystal, i.e. $x=$ $<100\rangle, y=<010\rangle$ and $z=<001\rangle$ (Case A). The analytical 
solution shows excellent agreement with the finite element numerical solution. The FEA solution is evaluated at the midplane, where generalized plane strain conditions prevail. It was observed that the stress field approaches the 2-D generaiized piane strain solution after a short distance from the edges, indicating that the analytical solution could be used effectively for many practical 3-D contact problems. The advantage of this analytical solution is that it is accurate, and extremely quick to compute, anywhere in the computational domain. The subsurface stress solutions are discussed in greater detail in later sections.

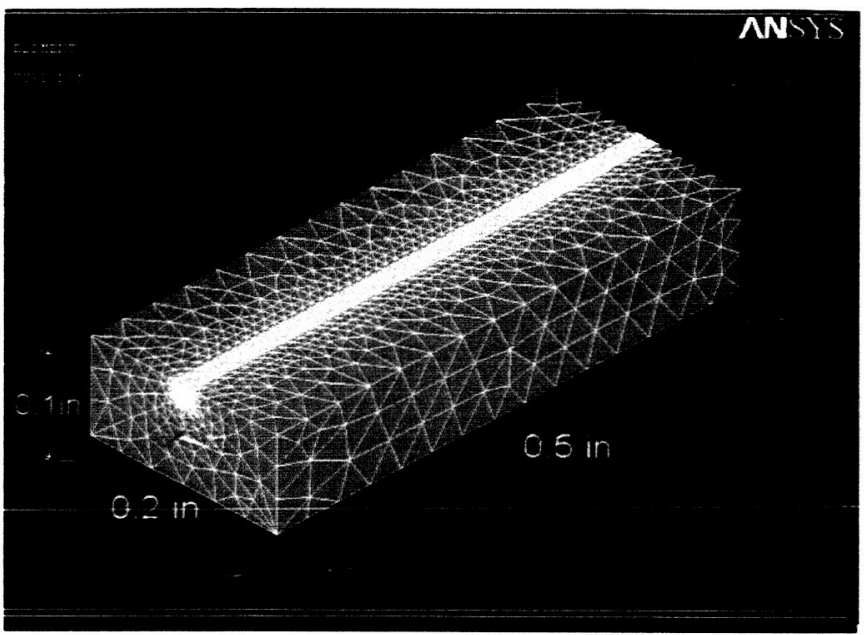

Figure 4. Three-Dimensional FE model of the elastic anisotropic half space [28]

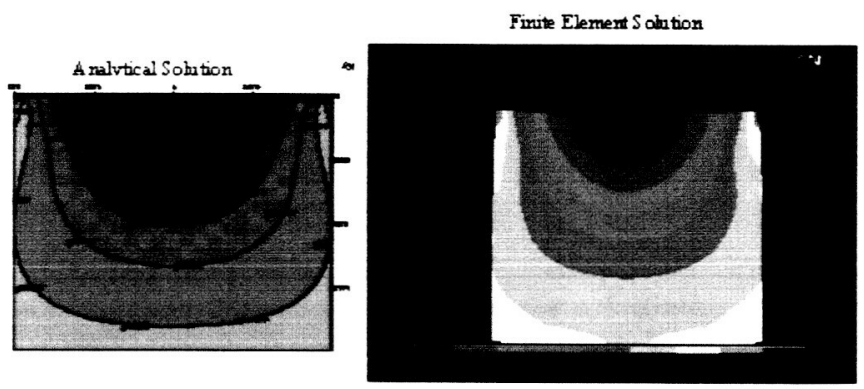

Figure 5. Stress $\left(\sigma_{\mathrm{y}}\right)$ contours using analytical solution and finite element (ANSYS) solution [28]

\section{Finite Element Analysis (FEA) of the Cylindrical Anisotropic Contact Problem}

A cylindrical indenter on an anisotropic half-space contact model (Figure 6) was developed in ANSYS. The cylindrical indenter was modeled using 4-node tetrahedral elements (SOLID45's) and the plate was modeled using 8-node hexahedral elements (SOLID45's) in the contact region and 4node tetrahedral elements (SOLID45's) in the far field. Surface-to-surface contact elements (CONTA174 and TARGE170) were used at the interface of the two bodies. Because of very high stress gradients in the contact region, a highly refined FE mesh must be used to obtain reliable stress solutions. The densely meshed regions in both the halfcylinder and half-space have roughly the same element size. The refined mesh and the iterative solution of the contact problem require computationally intensive resources, both in time and space. A typical analysis takes two CPU hours on a $2.4 \mathrm{GHz}$ multiprocessor PC-based workstation.

The analytical solution outlined in the previous section can be used for obtaining subsurface stresses in a half space for a known or applied normal and tangential tractions. The analytical solution was obtained by applying a semi-elliptical normal pressure distribution over the contact width. The contact width, $2 a$, was estimated initially using a Hertzian isotropic calculation. The FEA contact model converges to the correct anisotropic contact width. Figure 7 shows a representative comparison of subsurface stresses computed using the analytical solution and FEA contact model (Figure 6). Excellent agreement is seen between the two solutions.

The crystallographic orientations are designated by successive rotations about the $(X Y Z)$ axes, as follows: $\gamma$ is rotation about $\mathrm{X}$ axis, $\Delta$ is rotation about $Y$ ' axis, and $\theta$ is rotation about $Z$ ' axis. Table 2 shows 4 different crystallographic orientations considered in the analysis. Figure 8 shows some representative contour plots of the resolved shear stress values for slip systems $\tau_{1}, \tau_{3}$, and $\tau_{11}$, for two different crystallographic orientations: Case B $\left(\Delta=15^{\circ}, \gamma=0^{\circ}, \theta=0^{\circ}\right)$ and Case C $\left(\Delta=-15^{\circ}, \gamma=0^{\circ}, \theta=0^{\circ}\right)$. The plane on which the crack will nucleate will depend on the magnitude of the shear stress amplitude. Even though the contour plots show similarity between Cases B and C, the RSS values are very different, because of material orthotropy and the stresses are a strong function of both primary and secondary crystal orientation.
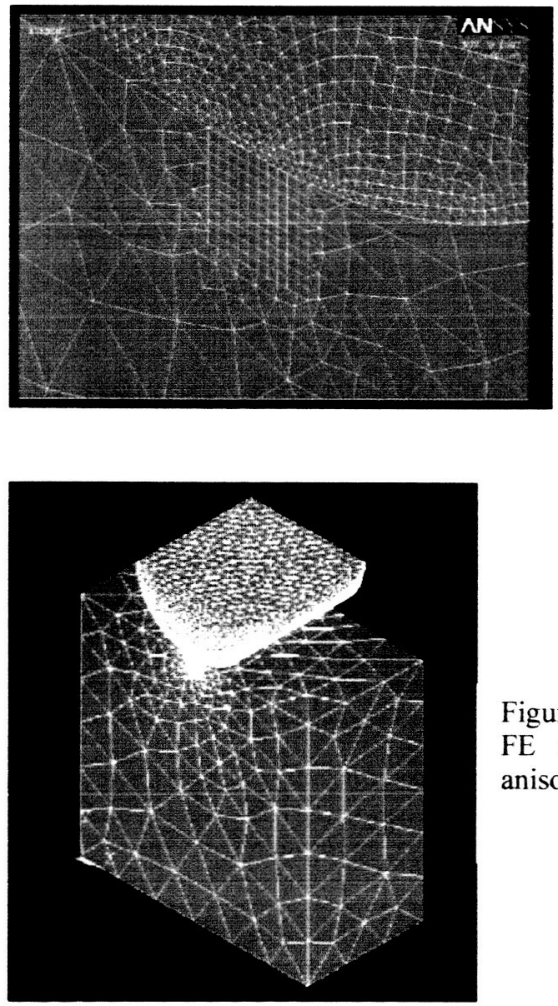

Figure 6. Three-dimensional FE model of a cylindrical anisotropic contact [28] 
Table 2 Designation of crystallographic orientations

\begin{tabular}{|c|c|c|c|}
\hline Case & $\Delta^{\circ}$ & $\gamma^{\circ}$ & $\theta^{\circ}$ \\
\hline $\mathrm{A}$ & 0 & 0 & 0 \\
\hline $\mathrm{B}$ & +15 & 0 & 0 \\
\hline $\mathrm{C}$ & -15 & 0 & 0 \\
\hline $\mathrm{D}$ & 0 & 0 & 40 \\
\hline
\end{tabular}

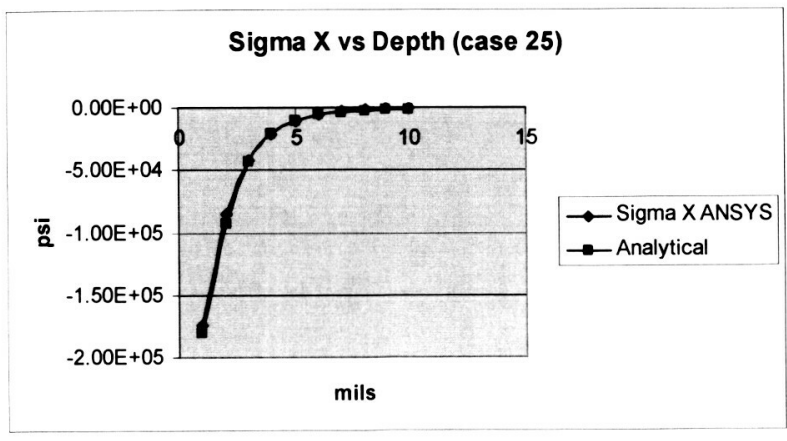

Sigma Y vs Depth (case 25)

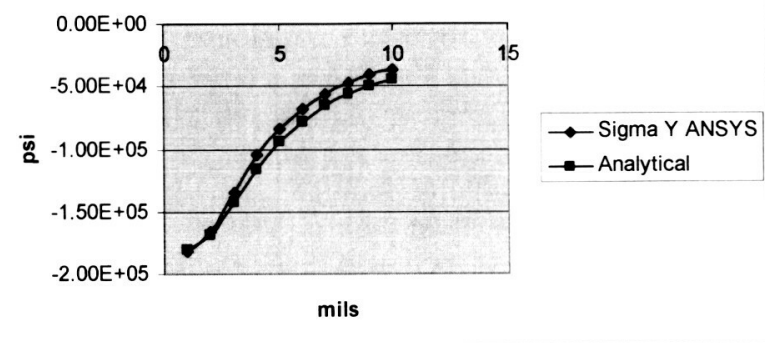

Figure 7. Comparison of FEA contact and analytical subsurface stresses $\sigma_{\mathrm{x}}$ and $\sigma_{\mathrm{y}}$, as a function of depth, for crystallographic orientation defined by Case $C\left(\Delta=-15^{\circ}, \gamma=0^{\circ}\right.$, $\theta=0^{\circ}$ ) [28]

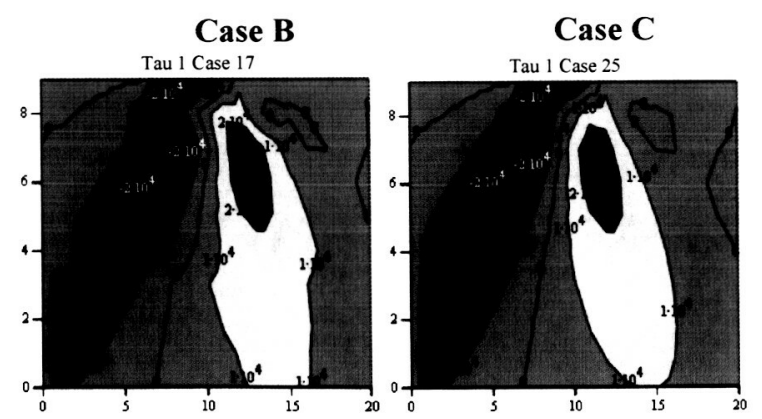

bb

bb
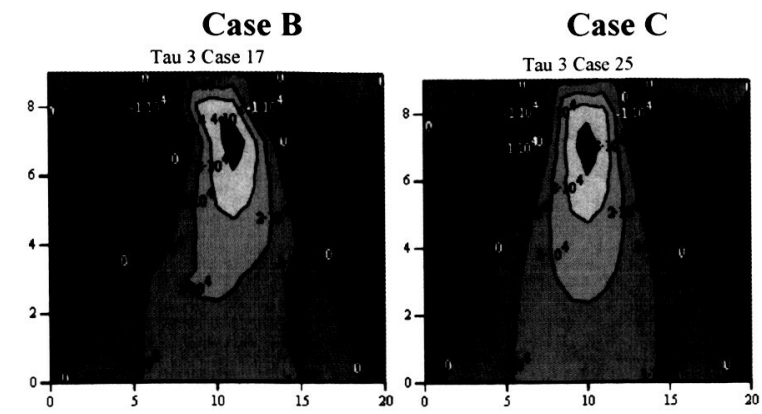

dd

Case B

Tau 11 Case 17

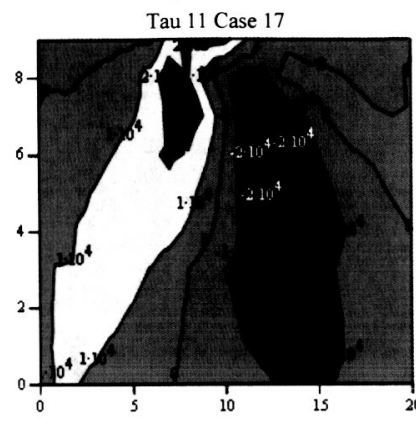

Case $\mathbf{C}$ Tau 11 Case 25

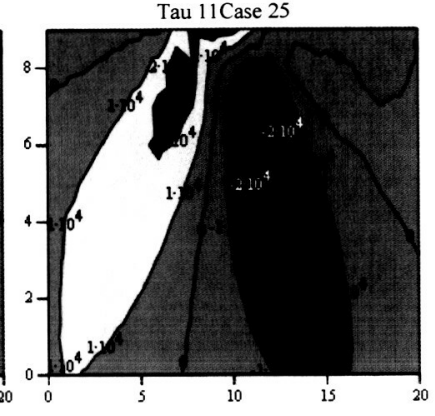

11

Figure 8. Contour plots of RSS $\tau_{1}, \tau_{3}$, and $\tau_{11}$, for Cases B and $\mathrm{C}$ under the contact region [28]

\section{Finite Element Analysis (FEA) of the Spherical Anisotropic Contact Problem}

A 3-D FEA of the spherical anisotropic contact problem was also performed using ANSYS. Figure 9 shows the FEA model of an isotropic spherical contact on a single-crystal plate. The sphere was modeled with 8-node hexahedral elements (SOLID45's) assuming linear-elastic isotropic material behavior. The plate was modeled with 20 -node hexahedral elements (SOLID95's) and 10-node tetrahedral elements (SOLID95's). Linear-elastic anisotropoic material properties were used in the plate. The contacting surface between the two bodies was represented using ANSYS surface-to-surface contact elements with friction (CONTA174-TARGE170). The indenter or damper is subjected to both normal and tangential loads and therefore frictional effects are incorporated.

An analytical solution for the 3-D anisotropic contact problem was also obtained using the stress function approach outlined in Lekhnitskii [1]. However, because of the complexity of the 3$\mathrm{D}$ analytical solution, its presentation is reserved for a separate article. The numerical results based on the FEA are reported herein. 


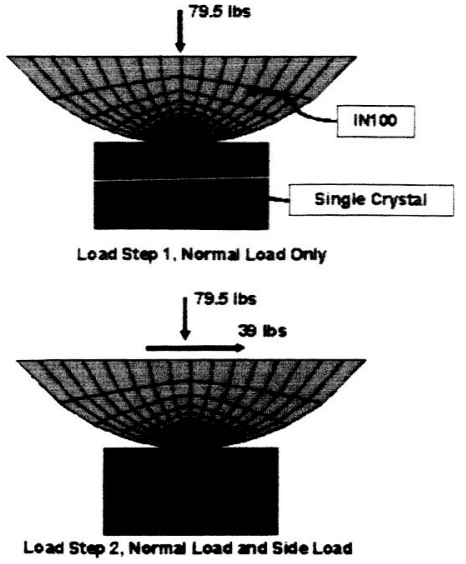

Figure 9. Three-dimensional FE model of the spherical isotropic indenter on a single crystal orthotropic substrate.

The Hertzian solution for a spherical isotropic contact on a flat plate is given by

$$
P=\frac{4 a^{3}}{3 R}\left(E^{*}\right) ; \quad E^{*}=\left(\frac{1-v_{1}^{2}}{E_{1}}+\frac{1-v_{2}^{2}}{E_{2}}\right) ; \quad p_{O}=\frac{1.5 P}{\pi a^{2}}
$$

where $E^{*}$ is the effective or composite modulus at the contact. To derive an effective modulus for the single crystal orthotropic contact we refer to J. R. Turner's paper [23]. We have adapted his work for a transversely isotropic contact to an orthotropic contact in question. The stress-strain relation in the material coordinate system is given by:

$$
E\left[\begin{array}{l}
\varepsilon_{x x} \\
\varepsilon_{y y} \\
\varepsilon_{z z} \\
\gamma_{x z} \\
\gamma_{y z} \\
\gamma_{x y}
\end{array}\right]=\left[\begin{array}{cccccc}
1 & -v_{H} & -v_{V} & & & \\
-v_{H} & 1 & -v_{V} & & & \\
-v_{V} & -v_{V} & \lambda & & & \\
& & & 2(1+v) & & \\
& & & & 2(1+v) & \\
& & & & & 2(1+v)
\end{array}\right]\left[\begin{array}{l}
\sigma_{x x} \\
\sigma_{y y} \\
\sigma_{z z} \\
\tau_{x z} \\
\tau_{y z} \\
\tau_{x y}
\end{array}\right]
$$

The solution for the orthotropic spherical contact can be derived as

$$
\begin{aligned}
& P=\frac{4 a^{3}}{3 R}\left(\frac{\pi^{2} \phi_{2} \kappa}{\phi_{1}}\right) \\
& E_{\text {ortho }}=\frac{\pi^{2} \phi_{2} \kappa}{\phi_{1}}
\end{aligned}
$$

where $P$ is the normal load, $a$ is the contact radius, $R$ is the sphere radius, and

$$
\begin{aligned}
& \phi_{1}=\frac{2 \gamma}{\sqrt{\alpha}-\gamma}, \quad \phi_{2}=\frac{2}{\varepsilon \pi(\sqrt{\alpha}-\gamma)} \\
& \alpha=\left(\frac{\lambda-v_{V}^{2}}{1-v_{H}^{2}}\right), \quad \kappa=\frac{1}{\pi} \ln \left(\frac{\sqrt{\alpha}+\gamma}{\sqrt{\alpha}-\gamma}\right) \\
& \gamma=\left(\frac{2}{\alpha+\beta}\right)^{1 / 2}\left(\frac{\alpha}{2}-\frac{v_{V}}{2\left(1-v_{H}\right)}\right) \\
& \varepsilon=\left(\frac{\alpha+\beta}{2}\right)^{1 / 2}\left(\frac{1-v_{H}}{G_{H}}\right)
\end{aligned}
$$

$E_{\text {ortho }}$ is an effective contact modulus that can be used to estimate the contact patch size, for the single crystal contact. For example, for crystal orientation Case $A\left(\Delta=0^{\circ}, \gamma=0^{\circ}, \theta=0^{\circ}\right)$, $R=0.25$ in and $P=79.5 \mathrm{lb}$, we can calculate a contact radius from Eq. (15) as $a_{\text {ortho }}=0.00814$ in. The contact radius calculated using FEA was $a_{\text {fea }}=0.0092$. The contact radius for the isotropic Hertzian calculation (Eq. 13) is $a_{\text {iso }}=0.0104$ in (based on $E_{1}=18.1 \mathrm{E} 06 \mathrm{psi}, E_{2}=31.2 \mathrm{E} 06 \mathrm{psi}, \mathrm{v}_{1}=0.3892, \mathrm{v}_{2}=$ 0.293 ). The effective modulus, $E_{\text {ortho }}$, is very useful for calculating the effective contact radius. Once the effective contact radius, $a_{\text {ortho }}$, is known, we can calculate the maximum contact pressure $p_{0}$. The semi-elliptic pressure distribution, $p_{o} \sqrt{1-r^{2}}$, can be applied as a normal pressure on the halfspace in the FEA, thus effectively decoupling the contact problem with the subsurface stress calculations and hence greatly simplifying the numerical problem.

\section{Case A}

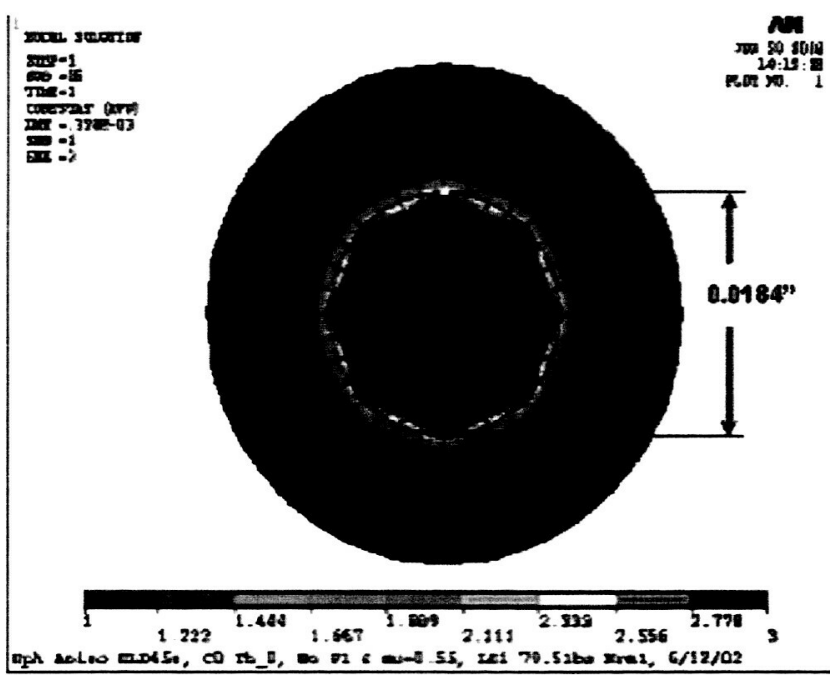


Case B

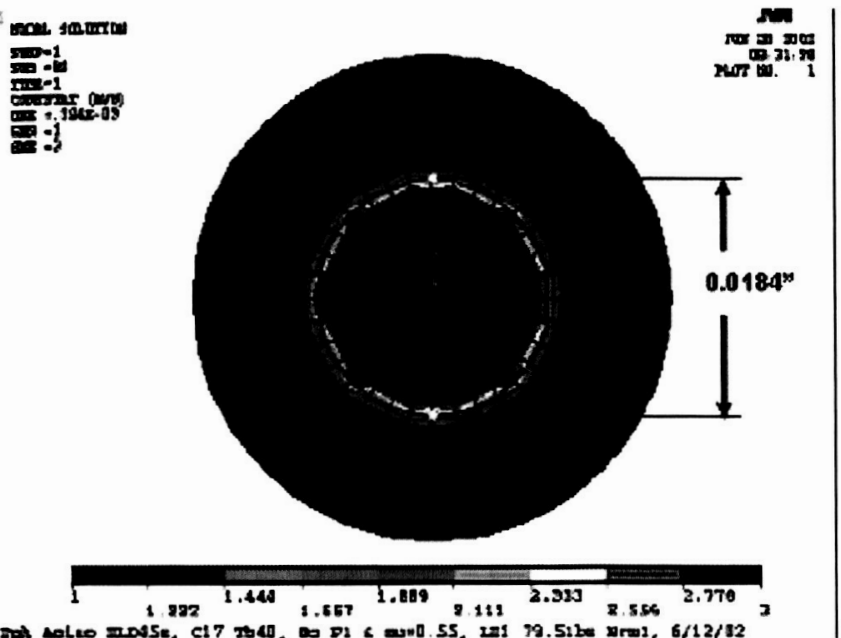

Figure 10. Spherical orthotropic contact radius as a function of crystal orientation

Figure 10 shows the contact patch calculated using FEA contact elements, for crystal orientation Cases A and D. Even though these two cases represent very different crystal orientations, the contact radius does not vary significantly from $a_{\mathrm{fea}}=0.0092$ in. However, it must be pointed out that the FEA mesh size in the contact region was 0.001 in square, and for better resolution the mesh size has to be refined, further highlighting the problems associated with FEA of anisotropic contact problems. Numerical accuracy issues in subsurface stresses as a function of mesh refinement in contact problems is discussed in detail by Beisham and Sinclair [29]. It is very advantageous to

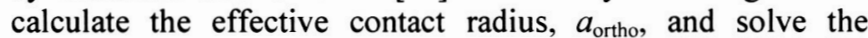
applied stress problem, rather than resorting to solving the problem using contact elements. This approach is the most effective way to solve contact problems involving single crystal substrates, especially for design iterations. This approach is illustrated in Figure 11.

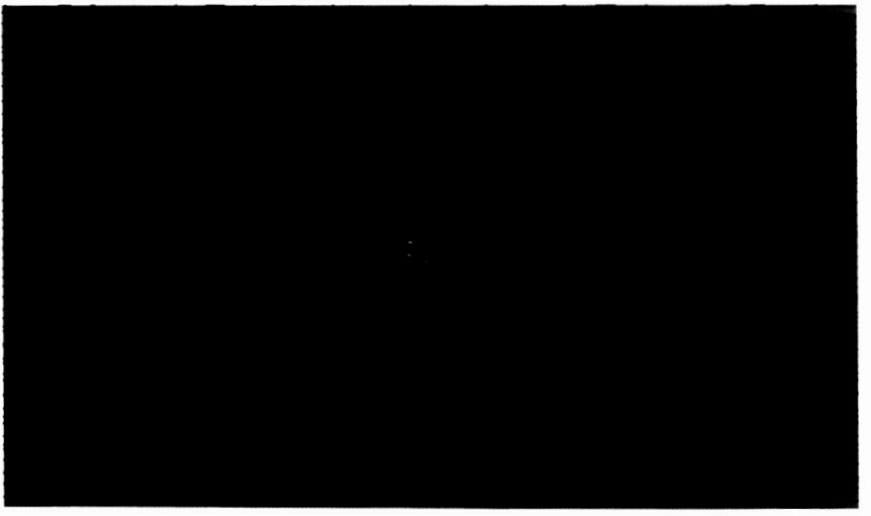

Figure 11. Decoupling the contact problem with subsurface stresses using the effective contact radius, $a_{\text {ortho }}$, using Eq. (15), and applying Hertzian normal loads over the contact patch
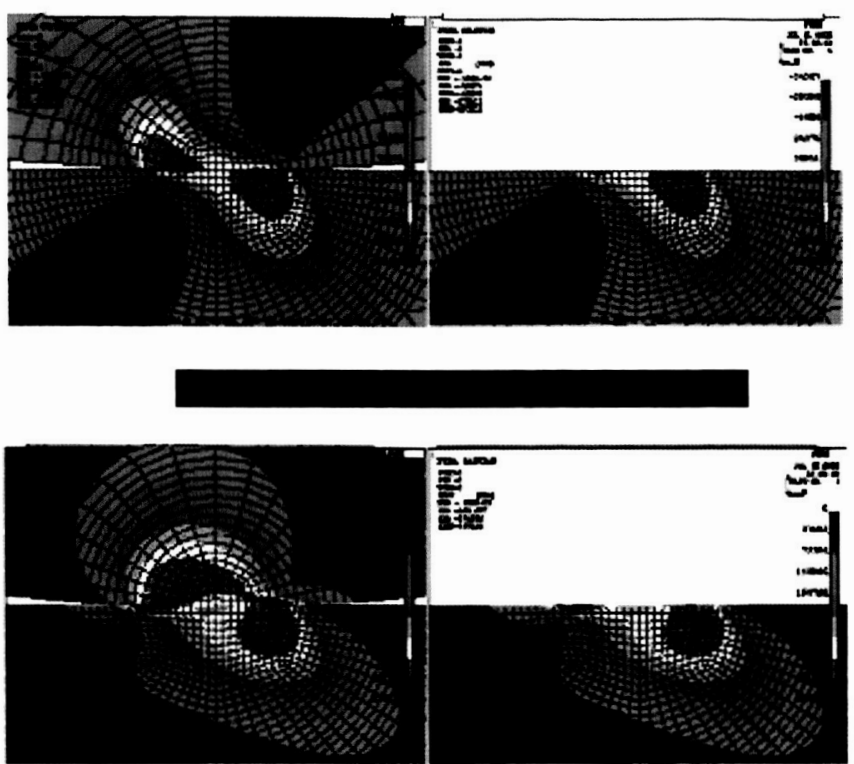

Figure 12. Comparison of subsurface stresses between the full FEA contact solution and simulated contact, for the orthotropic spherical contact

Representative subsurface stress results, using this decoupling approach and the full contact solution, are shown in Figure 12. Comparison shows excellent agreement between the two approaches.

Cylindrical and spherical contact simulations were performed for a wide range of crystallographic orientations. Normal contact pressure for these cases was compared with that of Case A $\left(\Delta=0^{\circ}, \gamma=0^{\circ}, \theta=0^{\circ}\right)$ where the coordinate axes are parallel to the crystal axes, to see the effect of crystal orientation. It was found that even for large orientation deviations from Case A, the normal contact pressure and contact patch size did not vary substantially, indicating that the effective contact modulus, $E_{\text {ortho, }}$ and contact width (cylindrical) or radius (spherical), $a_{\text {ortho, }}$ are relatively insensitive to variations in crystallographic orientation. The $E_{\text {ortho }}$ and $a_{\text {ortho }}$ values based on Case A orientation can be used for nearly all practical blade casting crystallographic orientation deviations from the ideal. This lends further credibility to using the simulated contact model for FEA, which leads to greatly simplified contact subsurface stress analysis. Even though the contact normal pressure does not change substantially, the subsurface stresses are a strong function of orientation. The simulated contact method is very advantageous for performing repeated subsurface stress calculations required for fatigue life calculations. The full contact solutions typically took 7-8 hours to run on a fast multiprocessor PC-based workstation, while the simulated contact took only 1-2 minutes to execute on the same machine.

Figure 13 shows the weighted percentage differences in contact pressure for some crystal orientations, as compared to Case A, for the spherical contact. It can be seen that the 

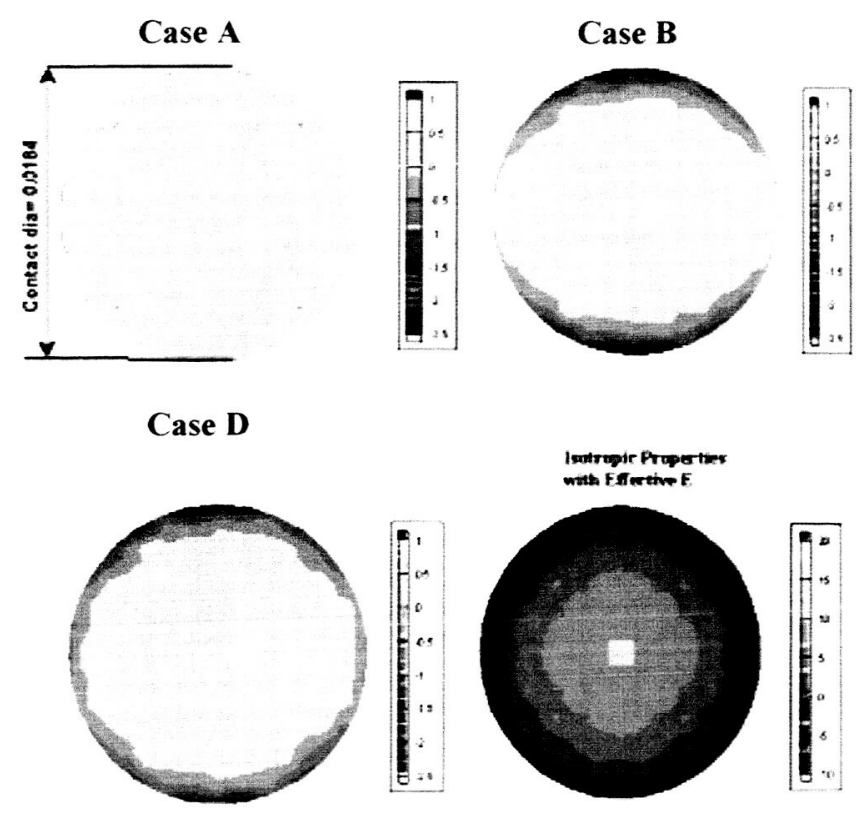

Figure 13. The weighted percentage difference in normal contact pressure for the orthotropic and isotropic spherical contact, as compared to Case A

deviation of normal pressure from Case A are within $2.5 \%$ for most practical situations. In contrast, the difference between the isotropic Hertzian contact (Eq. 13) with Case A is significantly higher (within $10 \%$ ).

\section{Fatigue Considerations}

The fatigue crack nucleation and crack growth behavior of single crystal nickel superalloys is governed by a complex interaction between the operative deformation mechanism, stress intensity, and environmental conditions. The fatigue crack growth behavior is determined by the operative microscopic fracture mode. As a result of the two-phase microstructure present in single crystal nickel alloys a complex set of fracture modes exist based on the dislocation motion in the matrix $(\gamma)$ and precipitate phase $(\gamma)$. A fatigue life model was obtained by Arakere and Swanson [9], based on strain controlled LCF tests conducted at $1200 \mathrm{~F}$ in air for single crystal uniaxial smooth specimens, for four different specimen orientations $\langle 001\rangle,\langle 111\rangle,\langle 213\rangle$ and $\langle 011\rangle$. Several multiaxial fatigue damage theories, including critical plane methods, were evaluated to identify a suitable fatigue damage parameter that would fit the test data well. The maximum shear stress amplitude, $\Delta \tau_{\max }$, on the slip systems was found to give the best fit for the test data, as shown in Figure 14. A power law curve fit for the data shown in Figure 14 was used as a fatigue life estimation equation $\left(1200^{\circ} \mathrm{F}\right)$, given below.

$$
\Delta \tau_{\max }=397,758 \mathrm{~N}^{-0} 1598
$$

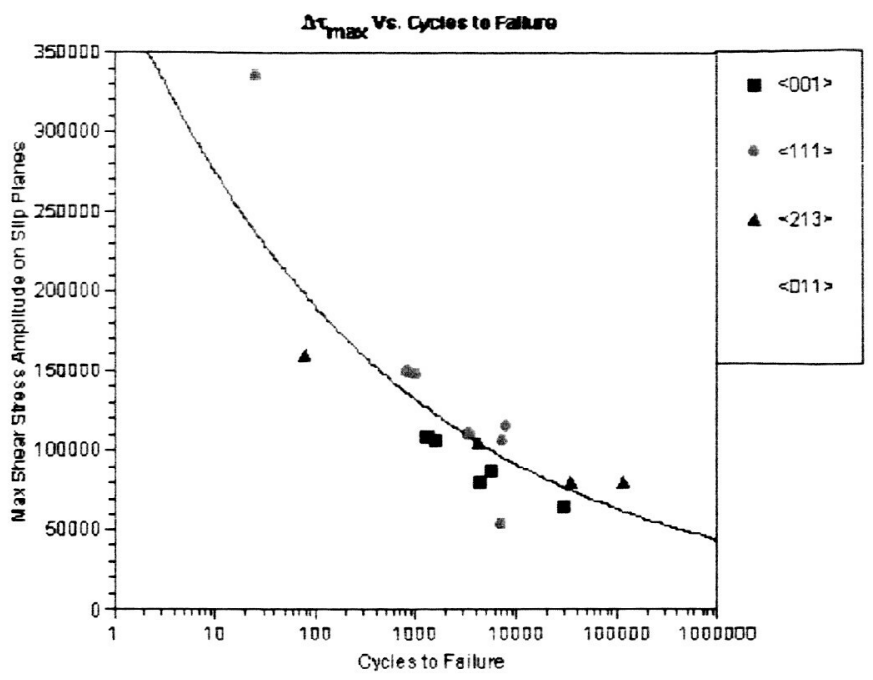

Figure 14. Fatigue damage parameter, $\Delta \tau_{\max }$ vs. Cycles to failure [9]

The fatigue damage parameter, $\Delta \tau_{\max }$, has been tested for an extensive set of single crystal fatigue data, under a range of environmental conditions, and was found to be effective [9, 11]. A cylindrical or spherical indenter contacting a single crystal substrate subject to a vibratory normal and tangential load will result in subsurface cyclic fatigue stresses. These fatigue stresses can lead to subsurface crystallographic cracks, as shown in Figure 2. Figure 8 shows the contour plots of RSS on the primary octahedral slip systems, for a cylindrical contact loaded with static normal and tangential loads. If the tangential loads are cycled, as would happen during fretting fatigue loading, we can compute the shear stress amplitudes $\Delta \tau_{1}$, $\Delta \tau_{2}, \ldots \ldots, \Delta \tau_{12}$, in the subsurface region. The subsurface location that yields the maximum $\Delta \tau$ value is likely to initiate a crystallographic fatigue crack.

We consider a critical subsurface location near the leading edge of contact, as shown in Figure 15. We will consider the situation where the tangential traction force, $q(x)$, is cycled between a positive and a negative value, and compute the shear stress amplitudes $\Delta \tau$ on the primary planes. Because the secondary crystallographic orientation is not controlled during the blade casting process, the variation in $\Delta \tau$ due to the variation in secondary orientation alone is of interest. This effect is illustrated in Figure 16. We see that maximum $\Delta \tau$ ( $\Delta \tau_{6}$, and $\Delta \tau_{11}$ in this case) values vary by $32 \%$ purely because of variation in secondary crystal orientation between $0^{\circ}$ to $90^{\circ}$. This can result in an order of magnitude variation in fatigue life calculated from Figure 14. 


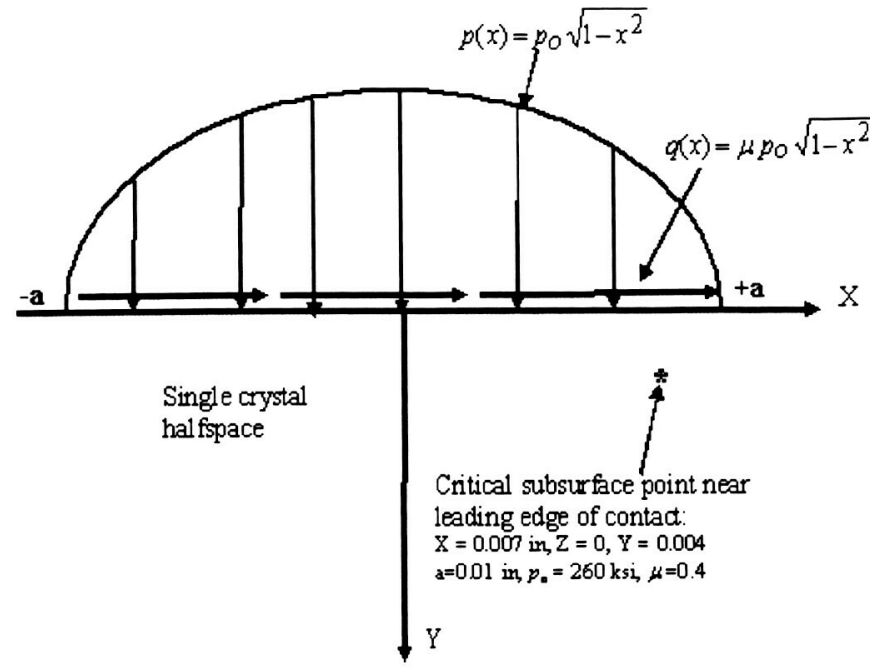

Figure 15. A critical subsurface point near the leading edge, for a cylindrical single crystal contact of width $2 a$.

Variation of $\Delta \tau$ is even greater at $\sim 65 \%$, due to a $15^{\circ}$ primary axis tilt from Case A. This can mean large variations in fatigue life between different blades, under the same loading conditions, as a result of blade-to-blade variations in primary and secondary crystallographic orientation.

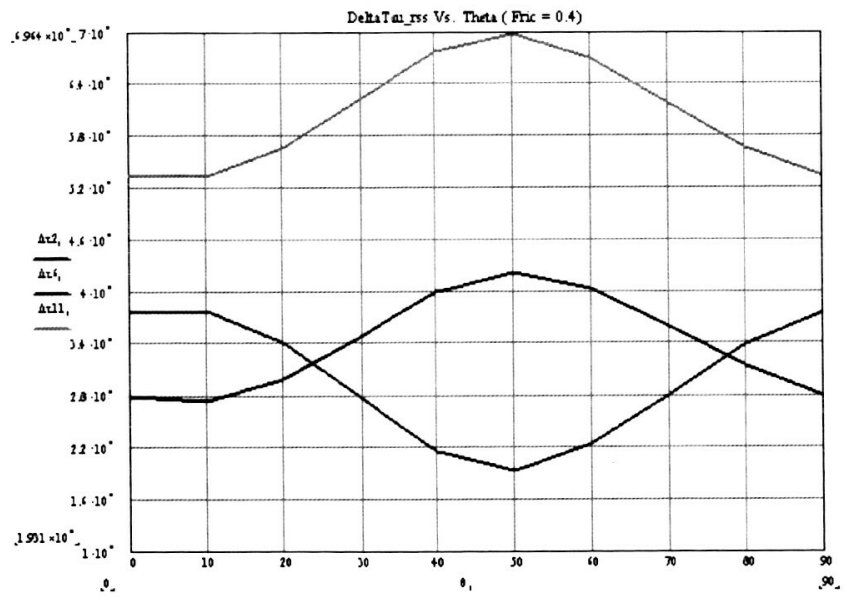

Figure 16. Variation of $\Delta \tau$ at the critical point shown in Figure 15 as a function of secondary crystallographic orientation $\theta$. ( primary orientation $=$ Case $\mathrm{A}$ )

\section{Conclusions}

A detailed evaluation of subsurface stresses in cylindrical and spherical orthotropic FCC single crystal nonconformal contacts is presented, using analytical and numerical techniques. Effects of variation in primary and secondary crystallographic orientation on subsurface stresses are included. Evaluation of subsurface stresses is an essential part of contact fatigue life calculations at damper contacts and dovetail attachment regions. A two-dimensional analytical solution for subsurface stresses in cylindrical single crystal contacts is presented, based on an adaptation of a stress function approach by Lekhnitskii [1]. Lekhnitskii's method for an anisotropic half space in generalized plane deformation has been adapted to a FCC orthotropic half space. The analytical solution showed excellent agreement with the 3-D FEA results. It was observed that the 3D FEA stress field approaches the 2-D generalized plane strain solution after a short distance from the edges, indicating that the analytical solution could be used effectively for many practical 3-D contact problems. The advantage of the analytical solution is that it is accurate, and extremely quick to compute, anywhere in the computational domain.

Three-dimensional FEA results for the spherical single crystal contact are presented. An effective contact modulus for the single crystal half space, $E_{\text {ortho }}$, and contact radius, $a_{\text {ortho }}$, (Eq. $15)$, is shown to be effective in calculating the contact patch size. The FEA of the contact problem can be greatly simplified by using $a_{\text {ortho }}$ and applying the normal pressure based on Hertzian assumptions over the contact patch. It is demonstrated that this applied stress problem, yields accurate subsurface stresses and greatly simplifies the FEA by avoiding the use contact elements. For a fixed normal load, the, $E_{\text {ortho }}$ and $a_{\text {ortho }}$ values were found to be relatively insensitive to variations in crystallographic orientation. Hence the $E_{\text {ortho }}$ and $a_{\text {ortho }}$ values based on Case A, $(\theta=0)$ orientation can be used for nearly all practical blade casting crystallographic orientation deviations from the ideal. This lends further credibility to using the simulated contact model for FEA. The simulated contact approach is very advantageous for performing repeated subsurface stress calculations required for fatigue life evaluation.

It must be noted that even though the contact area and normal pressure does not vary substantially with crystal orientation, the subsurface stresses are a strong function of orientation. Therefore the resolved shear stresses on the slip systems, and hence fatigue life, are a strong function of crystallographic orientation. It is shown that there can be an order of magnitude variation in contact fatigue life between different blades under the same loading conditions, as a result of blade-to-blade variations in primary and secondary crystallographic orientation.

Obtaining accurate subsurface stress results for anisotropic single crystal contact problems require extremely refined 3-D finite element grids, especially in the edge of contact region. Obtaining resolved shear stresses on principal slip planes also involves considerable post-processing work. For these reasons it is very advantageous to develop analytical solution schemes for subsurface stresses, whenever possible.

\section{ACKNOWLEDGMENTS}

The authors would like to acknowledge the NASA/ASEE Summer Faculty Fellowship Program and the Graduate Student Accompanying Program. The support from this program, administered by the University of Alabama in Tuscaloosa, enabled Nagaraj K. Arakere and Erik C. Knudsen to work during Summer 2002 at the NASA Marshall Space Flight 
Center, Huntsville, AL, where a majority of this work was completed.

\section{REFERENCES}

1. Lekhnitskii, S.G., Theory of Elasticity of an Anisotropic Elastic Body, San Francisco: Holden-Day, Inc., 1963, pp. $1-40$.

2. D. A. Hills and D. Nowell, Mechanics of Fretting Fatigue, Kluwer, Deventer, 1994.

3. J. Dombromirski, Variables of Fretting Process: Are There 50 of them? Standardization of Fretting Fatigue Test Methods and Equipment, ASTM, pp. 60-68, 1990.

4. B. A. Cowles, High cycle fatigue in aircraft gas turbinesan industry perspective, International Journal of Fracture, (1996) 1-16

5. Deluca, D., Annis. C, "Fatigue in Single Crystal Nickel Superalloys"; Office of Naval Research, Department of the Navy FR23800, August 1995.

6. C.T. Sims, "Superalloys: Genesis and Character", Superalloys - II, Eds., C.T. Sims, N.S. Stoloff and W.C. Hagel, Wiley \& Sons, New York, New York, (1987), p. 1.

7. F.L. VerSnyder and R.W. Guard, "Directional Grain Structure for High Temperature Strength", Trans. ASM, $52,(1960)$, p. 485 .

8. M. Gell and D.N. Duhl, "The Development of Single Crystal Superalloy Turbine Blades", Processing and Properties of Advanced High-Temperature Materials, Eds., S.M. Allen, R.M. Pelloux and R. Widmer, ASM, Metals Park, Ohio, (1986), p. 41.

9. N. K. Arakere and G. Swanson, "Effect of Crystal Orientation on Fatigue Failure of Single Crystal Nickel Base Turbine Blade Superalloys," ASME Journal of Gas Turbines and Power, v 124, pp. 161-176, Jan 2002.

10. G. Swanson and N. K. Arakere, "Fatigue Failure of Single Crystal Nickel Base Turbine Blade Superalloys," NASA/TP-2000-210074.

11. N. K. Arakere, "High Temperature Fatigue Properties of Single Crystal Superalloys in Air and Hydrogen," Paper 01-GT-585, Proceedings of the ASME Turbo Expo 2000, June 4-7, New Orleans, LA.

12. N. K. Arakere and G. Swanson, "Analysis of Fretting Stresses in Single Crystal Ni-Base Turbine Blade Attachment Regions," ASME Journal of Tribology, Vol. 123, pp. 413-423, 2001.

13. Personal Communication with D. P. DeLuca, Pratt \& Whitney, East Hartford, CT.

14. A. E. Giannokopoulos, T. C. Lindley, and S. Suresh, "Aspects of Equivalence Between Contact Mechanics and Fracture Mechanics: Theoretical Connections and a Life-Prediction Methodology for Fretting-Fatigue," Acta Materialia, v 46, n 9, pp. 2955-2968, 1998.

15. M. P. Szolwinski and T. N. Farris, "Mechanics of Fretting Fatigue Crack Formation," Wear, v 198, 1996, pp. 93-107.

16. M. H. Attia and R. B. Waterhouse, Editors, Standardization of Fretting Fatigue Test Methods and Equipment, ASTM (04-011590-30), STP 1159, (1992)

17. D. W. Hoeppner, Mechanisms of Fretting Fatigue and Their Impact on Test Methods Development, Standardization of Fretting Fatigue Test Methods and Equipment, ASTM, pp. 23-32, 1990.

18. O. Vingsbo and D. Soderberg, On fretting maps, Wear, 126 (1988) 131-147.

19. C. Ruiz, P. H. B. Boddington and K. C. Chen, An investigation of fatigue and fretting in a dovetail joint. Experimental mechanics, 24 (3) (1984) 208-217.

20. K. L. Johnson, Contact Mechanics, Cambridge: Cambridge University Press; 1985, pp. 84-106.

Green and Zerna, Theoretical Elasticity, Oxford: Clarendon Press; 1954.

J. R. Willis, Hertzian Contact of Anisotropic Bodies, $J$. Mech. Phys. Solids, v 14, 1966, pp. 163-176.

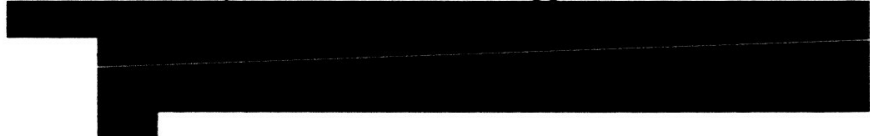

24. H. Fan and L. M. Keer, "Two-Dimensional Contact on an Anisotropic Half-Space", Trans of the ASME, v 61, 1994, pp. 250-255.

A. N. Stroh, "Dislocation and Cracks in Anisotropic Elasticity," Phil. Mag., Ser. 8, v 3, pp. 625-646.

26. J. J. Vlassak, et al., "The Indentation Modulus of Elastically Anisotropic Materials for Indenters of Arbitrary Shape," J. Mech. Phys. Solids v 51, 2003, pp. 1701-1721.

27. D. Stouffer and L. Dame, Inelastic Deformation of Metals: Models, Mechanical Properties, and Metallurgy, New York: John Wiley \& Sons, Inc.; 1996, pp. 387-417.

28. E. C. Knudsen, "Analytical and Numerical Evaluation of Subsurface Stresses in Anisotropic (Single-Crystal) Contacts," M. S. Thesis, 2003, Department of Mechanical \& Aerospace Engineering, University of Florida, Gainesville, Florida.

29. J. R. Beisheim and G. B. Sinclair, "Three-Dimensional Finite Element Analysis of Dovetail Attachments," Presented at the 2002 ASME Turbo Expo; Amsterdam, the Netherlands; June 3-6 2002. 